\title{
Numerical Study on Joint Quantization and Control under Block-Coding
}

\author{
Xiaobo Tan, Wei Xi, and John S. Baras
}

\begin{abstract}
This paper addresses the joint quantization and control problem for hidden Markov chains with variablelength block-coding. The aim is to understand the impact of communication bandwidth and information delay (due to block-coding) on the control performance. A heuristic algorithm is developed to solve the dynamic programming (DP) equation through the introduction of a metric on the discrete observation space. Numerical results are presented demonstrating the attention division in block-coding and the tradeoffs between control performance and communication bandwidth.
\end{abstract}

\section{INTRODUCTION}

Sensing and/or actuation signals in networked control systems are quantized and transmitted through networks for processing or execution. Understanding the impact of information compression is central to proper assignment of communication and computing resources in such systems. Control with communication constraints has received increasing attention in recent years, see, e.g., [1], [2], [3], [4], [5] and the references therein. In [6] the authors studied optimal sequential quantization for Markov sources. Optimal control of Markov systems (in particular, finite-state Markov chains) under communication constraints was discussed in [7]. There, again, sequential output quantization was considered; namely, the observation $Y_{n}$ is quantized and sent to the controller at each time $n$. It is well-known that, if one is only concerned about the compression error, vector quantization on long data blocks should be used [8]. For real-time control systems, the delay associated with blockcoding might pose a problem. But a more careful study is required to answer questions like, "can block-coding with moderate block lengths strike the best balance between coding efficiency and control performance?".

The problem of joint quantization and estimation with variable length block-coding was investigated in [9] for hidden Markov chains. Preliminary study on joint sequential quantization and control was also conducted in [9] with analysis on an example problem. In this paper the joint quantization and control problem is extended to the case where block-coding is allowed. To highlight the idea, it is

This research was supported by the Army Research Office under the ODDR\&E MURI01 Program Grant No. DAAD19-01-1-0465 to the Center for Networked Communicating Control Systems (through Boston University), and under ARO Grant No. DAAD190210319.

$\mathrm{X}$. Tan is with the Institute for Systems Research, University of Maryland, College Park, MD 20742, USA xbtan@umd.edu

W. $\mathrm{Xi}$ and J. S. Baras are with the the Institute for Systems Research and the Department of Electrical \& Computer Engineering, University of Maryland, College Park, MD 20742, USA $\{$ wxi, baras\}@glue.umd.edu assumed that data transmission is completed instantly and thus induces zero delay. But block-coding itself leads to times when the controller does not receive any data, in which case it still has to produce a control action based on the previous data and the system model.

The "curse of dimensionality" for dynamic programming (DP), coupled with the combinatorial nature of the problem, makes solving the DP equation a formidable task. A heuristic algorithm is introduced to approximately solve the DP, which provides significant speedup. This algorithm is based on the introduction of a metric on the discrete observation space. In the numerical examples the heuristic algorithm is shown to yield the same solutions as obtained through exhaustively searching the solution space.

Understanding the bits allocation in block coding is of interest for the development of efficient coding schemes. Based on the analysis of mutual information between the state and the observation, it is predicted that the more recent observation will be paid more attention (i.e., be quantized more finely) in block-coding. This prediction is verified by numerical results. Numerical computations are also performed to study the tradeoffs between control performance and communication bandwidth.

The remainder of the paper is organized as follows. In Section II the joint quantization and control problem with block-coding is formulated and a DP equation obtained. The heuristic scheme is described in Section III. Section IV discusses the attention division problem. Numerical results on the tradeoff study are presented in Section V. Some concluding remarks are provided in Section VI.

\section{Optimal Quantization And CONTROL With BLOCK-CODING}

Recall the setup in [9]. Consider a homogeneous, controlled, hidden Markov chain (HMC) $X_{n}$ taking values in $\mathcal{X}=\left\{x_{1}, \cdots, x_{S}\right\}$ for some $S \geq 1$. The control $U_{n}$ takes values in $\mathcal{U}=\left\{u_{1}, \cdots, u_{K}\right\}$ for some $K \geq 1$, and the output $Y_{n}$ takes values in $\mathcal{Y}=\left\{y_{1}, \cdots, y_{M}\right\}$ for some $M \geq 1$. For $u \in \mathcal{U}, 1 \leq i, j \leq S, 1 \leq k \leq M$, denote

$$
\begin{aligned}
a_{i j}(u) & \triangleq \operatorname{Pr}\left[X_{n+1}=x_{i} \mid X_{n}=x_{j}, U_{n}=u\right], \\
c_{i k} & \triangleq \operatorname{Pr}\left[Y_{n}=y_{k} \mid X_{n}=x_{i}\right] .
\end{aligned}
$$

The observation $Y_{n}$ needs to be compressed (with possibly block-coding) and transmitted to a remote controller. The controller then computes the control action $U_{n}$ based on the received bits and sends it back to the HMC. It is assumed that the communication link from the HMC to 
the controller is bandwidth-limited, while the link from the controller to the HMC has no bandwidth limitation (such asymmetry happens, e.g., when the remote site has much more power for communication than the HMC does).

Let $\Pi_{0}=\left(\pi_{0}\left(x_{1}\right), \cdots, \pi_{0}\left(x_{S}\right)\right)$ be the a priori probability mass function (PMF) for $X_{0}$. Given $N>0$, the goal is to find an admissible (to be made precise below) quantization and control decision $\delta$ such that the following cost is minimized:

$$
J\left(\Pi_{0}, \delta\right)=E\left[\sum_{n=0}^{N-1} \lambda J^{c}(n)+J^{r}(n)\right],
$$

where $J^{c}(n)$ is the communication cost at time $n, J^{p}(n)=$ $g_{n}\left(X_{n}, U_{n}\right)$ is the running cost at $n$, and $\lambda$ is a weighting coefficient. By varying $\lambda$, one could obtain a family of optimal solutions reflecting different tradeoffs between the control performance and the communication consumption.

At time 0 one determines the length of the first data block $Y_{0}^{n_{1}}=\left(Y_{0}, Y_{1}, \cdots, Y_{n_{1}}\right)$ and the quantization scheme $\mathcal{Q}(\cdot)$ for this block. Here $n_{1}+1 \leq B$ (the maximum block length). Control actions $U_{0}^{n_{1}}$ should also be specified appropriately at time 0 . At time $n_{1}$ the quantized information $q_{n_{1}}=\mathcal{Q}\left(Y_{0}^{n_{1}}\right)$ is sent to the controller. Note that if a plain binary coding is used (as in the rest of the paper),

$$
J^{c}(n)=\left\{\begin{array}{l}
0 \text { if } 0 \leq n<n_{1} \\
\log _{2}\left(\left|q_{n_{1}}\right|\right) \text { if } n=n_{1}
\end{array},\right.
$$

where $\left|q_{n_{1}}\right|$ denotes the number of possible outcomes of $q_{n_{1}}$. At $n_{1}$, one also needs to plan the quantization and control decision for the next data block. This process continues until it reaches $N-1$. Time instants such as $0, n_{1}, \cdots$, are called decision times. Denote by $R_{n}$ the bits received by the controller before time $n$. A quantization/control decision is admissible if:

- The quantization decision at time $n$ (if $n$ is a decision time) for the next data block depends only on $\Pi_{0}, R_{n}$, and $U_{0}^{n-1}$;

- $U_{n}$ depends only on $\Pi_{0}, U_{0}^{n-1}, R_{n}$, and $q_{n}$ (if any information is sent at $n$ ).

This is an "equi-memory" condition [7], and it eliminates the need to transmit the quantization/control decision separately. On the other hand, it entails certain storage/computation capability at the HMC side; in particular, it needs to store the optimal quantization and control policies (computed off-line), update the information state, and perform online table lookup for quantization decisions.

For $1 \leq j \leq B$, let $\mathcal{Y}^{j}$ be the product space of $j$ copies of $\mathcal{Y}$. Let $\Theta_{j}$ be the space of quantization (encoding) schemes for a data block of length $j$, i.e., $\forall \mathcal{Q}_{j} \in \Theta_{j}, \mathcal{Q}_{j}: \mathcal{Y}^{j} \rightarrow$ $\left\{1,2, \cdots, M^{j}\right\}$. Note that each $\mathcal{Q}_{j}$ is essentially a partition scheme for $\mathcal{Y}^{j}$. Denote by $\hat{\Pi}_{i}=\left\{\hat{\pi}_{i}\left(x_{1}\right), \cdots, \hat{\pi}_{i}\left(x_{S}\right)\right\}$ the conditional PMF of $X_{i}$ given $\Pi_{0}, R_{i}$, and $U_{0}^{i-1}$. One can write down a recursive formula for $\hat{\Pi}_{i}$; in particular, for $\mathcal{Q}_{j} \in \Theta_{j}$,

$$
\hat{\Pi}_{i+j}=F_{j}\left(\hat{\Pi}_{i}, \mathcal{Q}_{j}\left(Y_{i}^{i+j-1}\right), U_{i}^{i+j-1}\right),
$$

for some function $F_{j}$ (An example of $F_{j}$ for $j=1$ can be found in [9]).

Now define a sequence of optimal control problems with the cost functions $J_{i} ; 0 \leq i \leq N-1$, where $J_{i}$ is obtained by replacing " $n=0$ " in (1) with " $n=i$ ". Let the value function of the $i$-th problem be $V_{i} \cdot\left\{V_{i}\right\}_{i=0}^{N-1}$ can be solved by DP.

Proposition 2.1: Let $\Pi_{i}$ be the initial condition (a priori $\mathrm{PMF}$ ) of the $i$-th problem. For $1 \leq j \leq B, \mathcal{Q}_{j} \in \Theta_{j}$, denote by $\left|\mathcal{Q}_{j}\right|$ the number of partitions under $\mathcal{Q}_{j}$, and by $\mathcal{A}_{\mathcal{Q}_{j}}$ the space of functions mapping the range of $\mathcal{Q}_{j}$ to $\mathcal{U}$. The value functions $V_{i} ; 0 \leq i \leq N-1$, satisfy:

$$
\begin{aligned}
V_{N-1}\left(\Pi_{N-1}\right) & =\min _{\mathcal{Q}_{1} \in \Theta_{1}} \min _{\alpha \in \mathcal{A}_{\mathcal{Q}_{1}}} \lambda \log _{2}\left(\left|\mathcal{Q}_{1}\right|\right) \\
+ & E\left[g_{N-1}\left(X_{N-1}, \alpha\left(\mathcal{Q}_{1}\left(Y_{N-1}\right)\right)\right)\right]
\end{aligned}
$$

and for $0 \leq i \leq N-2$,

$$
\begin{array}{r}
V_{i}\left(\Pi_{i}\right)=\min _{j \in\{1, \cdots, B\}} \min _{\mathcal{Q}_{j} \in \Theta_{j}} \lambda \log _{2}\left(\left|\mathcal{Q}_{j}\right|\right)+ \\
\min _{v_{0}, \cdots, v_{j-2} \in \mathcal{U}} \min _{\alpha \in \mathcal{A}_{\mathcal{Q}_{j}}} E\left[\sum_{k=0}^{j-2} g_{i+k}\left(X_{i+k}, v_{k}\right)\right. \\
+g_{i+j-1}\left(X_{i+j-1}, \alpha\left(\mathcal{Q}_{j}\left(Y_{i}^{i+j-1}\right)\right)\right)+ \\
V_{i+j}\left(F_{j}\left(\Pi_{i}, \mathcal{Q}_{j}\left(Y_{i}^{i+j-1}\right), v_{0}^{j-2}, \alpha\left(\mathcal{Q}_{j}\left(Y_{i}^{i+j-1}\right)\right)\right)\right] .
\end{array}
$$

Sketch of proof. Note that there exist at most $B$ choices for the block length, and then use the dynamic programming principle.

By properly concatenating the minimizers in (3) and (4), one obtains the optimal quantization and decision scheme. Note that at any decision instant $n$, based on the current information state $\hat{\Pi}_{n}$, one decides the optimal block length $j^{*}$, the associated quantization scheme $\mathcal{Q}_{j^{*}}^{*}$, the controls $U_{n}^{n+j^{*}-2}$ (since no observation is available at these times), and the control map $\alpha^{*}$ at time $n+j^{*}-1$.

\section{A Heuristic SCHEME}

Solving the DP equations (3) and (4) exactly is prohibitive even for small-size problems due to their combinatorial nature. A heuristic scheme is developed here to trade some accuracy for computational speed. The key observation is that, the output (or the quantized output) provides useful information only through the conditional PMF of the state. For each block length $j$, instead of exhaustively searching over the whole space $\Theta_{j}$ for quantizing $Y_{i}^{i+j-1}$, in this scheme one only clusters outcomes of $Y_{i}^{i+j-1}$ that yield "adjacent" a posteriori PMFs. The underlying rationale is that, if the conditional PMFs $\hat{\Pi}^{(1)}$ and $\hat{\Pi}^{(2)}$ corresponding to two outcomes $\mathbf{y}^{(1)}$ and $\mathbf{y}^{(2)}$ are approximately equal, then the resulting conditional PMF given observing either $\mathbf{y}^{(1)}$ or $\mathbf{y}^{(2)}$ (i.e., $\mathbf{y}^{(1)}$ and $\mathbf{y}^{(2)}$ are grouped together) is

$$
\frac{\operatorname{Pr}\left(\mathbf{y}^{(1)}\right)}{\operatorname{Pr}\left(\mathbf{y}^{(1)}\right)+\operatorname{Pr}\left(\mathbf{y}^{(2)}\right)} \hat{\Pi}^{(1)}+\frac{\operatorname{Pr}\left(\mathbf{y}^{(2)}\right)}{\operatorname{Pr}\left(\mathbf{y}^{(1)}\right)+\operatorname{Pr}\left(\mathbf{y}^{(2)}\right)} \hat{\Pi}^{(2)},
$$


which is approximately equal to $\hat{\Pi}^{(1)}$ and $\hat{\Pi}^{(2)}$. Therefore, the information loss due to quantization (clustering) is small.

To further illustrate the idea, consider a one-stage problem $(N=1)$ with the number of states $S=2$. In this case it suffices to consider only the first component $\hat{\pi}_{0}\left(x_{1}\right)$ of $\hat{\Pi}_{0}$.

Proposition 3.1: Let $N=1, S=2$, and $M \geq 3$. Let $\left(\mathcal{Q}_{1}^{*}, \alpha^{*}\right)$ be an optimal quantization/control scheme. For $1 \leq k \leq 3$, let $y^{(k)} \in \mathcal{Y}$, and denote

$$
\hat{\pi}^{(k)}=\operatorname{Pr}\left(X_{0}=x_{1} \mid Y_{0}=y^{(k)}\right) .
$$

If

$$
\hat{\pi}^{(1)}<\hat{\pi}^{(2)}<\hat{\pi}^{(3)},
$$

then the following cannot happen:

$$
\mathcal{Q}_{1}^{*}\left(y^{(1)}\right)=\mathcal{Q}_{1}^{*}\left(y^{(3)}\right) \neq \mathcal{Q}_{1}^{*}\left(y^{(2)}\right) .
$$

Proof. Fix a quantization level $L$ (number of clusters), $1 \leq L \leq M$. We shall show that among all $\mathcal{Q}_{1}$ with $\left|\mathcal{Q}_{1}\right|=$ $L$, the one minimizing the running cost $E\left[g_{0}\left(X_{0}, U_{0}\right)\right]$ does not satisfy (6).

Let $\pi=\operatorname{Pr}\left(X_{0}=x_{1} \mid\right.$ (quantized) observation). Fix $u \in$ $\mathcal{U}$

$$
E_{\pi}\left[g_{0}\left(X_{0}, u\right)\right]=\pi g\left(x_{1}, u\right)+(1-\pi) g\left(x_{2}, u\right),
$$

which is a linear function of $\pi$. Minimizing (7) with respect to $u$, one gets a concave function $h(\pi)=$ $\min _{u \in \mathcal{U}} E_{\pi}\left[g\left(X_{0}, u\right)\right]$.

For ease of discussion, assume that $M=3$ (the case $M>3$ can be dealt with similarly). To further simplify the presentation, assume that $\operatorname{Pr}\left(y^{(1)}\right)=\operatorname{Pr}\left(y^{(2)}\right)=$ $\operatorname{Pr}\left(y^{(3)}\right)=\frac{1}{3}$ (Note $\operatorname{Pr}\left(y^{(k)}\right)=\operatorname{Pr}\left(Y_{0}=y^{(k)}\right)$ given the a priori $\left.\Pi_{0}\right)$. Consider $L=2$, and there are three possible clustering schemes: a) $\left[y^{(1)}, y^{(2)}\right],\left[y^{(3)}\right]$; b) $\left[y^{(1)}\right],\left[y^{(2)}, y^{(3)}\right]$; c) $\left[y^{(1)}, y^{(3)}\right],\left[y^{(2)}\right]$. Denote the running costs corresponding to the schemes by $J^{(a)}, J^{(b)}$, and $J^{(c)}$, respectively. For the equi-probability case here, we shall prove $J^{(a)} \leq J^{(c)}$ and $J^{(b)} \leq J^{(c)}$; for general cases, one can prove $\min \left(J^{(a)}, J^{(b)}\right) \leq J^{(c)}$. To show $J^{(a)} \leq J^{(c)}$, denote $\pi^{\prime}=\frac{\pi^{(1)}+\pi^{(2)}}{2}$ and $\pi^{\prime \prime}=\frac{\pi^{(1)}+\pi^{(3)}}{2}$. Then

$$
J^{(a)}=\frac{2 h\left(\pi^{\prime}\right)}{3}+\frac{h\left(\pi^{(3)}\right)}{3}, J^{(c)}=\frac{2 h\left(\pi^{\prime \prime}\right)}{3}+\frac{h\left(\pi^{(2)}\right)}{3} .
$$

Since $\pi^{\prime}<\pi^{\prime \prime}<\pi^{(3)}$, and $\pi^{\prime}<\pi^{(2)}<\pi^{(3)}$, by the concavity of $h(\cdot)$,

$$
\frac{h\left(\pi^{\prime \prime}\right)-h\left(\pi^{\prime}\right)}{\pi^{\prime \prime}-\pi^{\prime}} \geq \frac{h\left(\pi^{(3)}\right)-h\left(\pi^{(2)}\right)}{\pi^{(3)}-\pi^{(2)}},
$$

implying $J^{a} \leq J^{c}$. Similarly $J^{(b)} \leq J^{(c)}$ is proved.

The study of extension of Proposition 3.1 to the case $N \geq 2$ is underway. The above proposition suggests that in quantization (for the purpose of control) one may use metrics on the space of PMFs to measure the "distance" between (discrete) output outcomes. Based on such a metric many quantization schemes can be ruled out without detailed evaluation, which provides computational savings.

For $S=2$, the heuristic is particularly simple since only one component of $\hat{\Pi}_{0}$ is needed and it becomes a one-dimensional problem. Table I compares the number $N_{q}$ of quantization schemes to be evaluated under exhaustive search with that under the heuristic method, where $N_{o}$ denotes the number of possible outcomes (e.g., for a data block of length 2, $N_{o}=M^{2}$ ). It is clear from the table that the exhaustive search soon becomes unaffordable, and the heuristic provides a feasible alternative.

TABLE I

COMPLEXITY COMPARISON BETWEEN THE EXHAUSTIVE SEARCH AND THE HEURISTIC ALGORITHM.

\begin{tabular}{|c|c|c|}
\hline$N_{O}$ & $N_{q}$ (Exhaustive) & $N_{q}$ (Heuristic) \\
\hline 5 & 52 & 16 \\
\hline 7 & 877 & 64 \\
\hline 9 & 21,147 & 256 \\
\hline 11 & 678,570 & 1,024 \\
\hline 13 & $27,644,437$ & 4,096 \\
\hline 15 & $1,382,958,545$ & 16,384 \\
\hline
\end{tabular}

Fig. 1 compares the value functions obtained through the exhaustive search and the approximation method. Here the horizontal axis is the first component of a priori PMF for $X_{0}$. In the computation $N=4, B=1, S=2, M=5$, $\lambda=0.01, A(u)=\left[a_{i j}(u)\right]$ and $C=\left[c_{i k}\right]$ are given by

$$
\begin{gathered}
A\left(u_{1}\right)=\left[\begin{array}{ll}
2 / 3 & 0 \\
1 / 3 & 1
\end{array}\right], A\left(u_{2}\right)=\left[\begin{array}{ccc}
2 / 3 & 2 / 3 \\
1 / 3 & 1 / 3
\end{array}\right], \\
C=\left[\begin{array}{lllll}
1 / 15 & 2 / 15 & 3 / 15 & 4 / 15 & 5 / 15 \\
5 / 15 & 4 / 15 & 3 / 15 & 2 / 15 & 1 / 15
\end{array}\right],
\end{gathered}
$$

and the cost matrix $G=\left[g_{i j}\right]$, where $g_{i j}=g_{n}\left(X_{n}=\right.$ $\left.x_{i}, U_{n}=u_{j}\right), \forall n$, is

$$
G=\left[\begin{array}{ll}
0 & 1 \\
2 & 1
\end{array}\right] .
$$

For this example the two value functions overlap and the heuristic algorithm yields the exact solution. In another computed example (not shown here) with $B=2$ and $M=3$, the solutions generated by the heuristic again agree with those by the exhaustive search.

\section{ATtention Division In Block-Coding}

It is of interest to study the attention division problem in block-coding. For example, if a block $Y_{i}^{i+j-1}$ of length $j$ is quantized, one can ask how the bits are divided among $Y_{n} ; i \leq n \leq i+j-1$. Answers to such questions can help in evaluating the importance of different data samples, and may provide hint in developing approximate solutions.

Note that in (4), the quantized version of $Y_{i}^{i+j-1}$ is used for evaluation of the information state at time $i+j-1$, which is subsequently used for picking control action at $i+j-1$ and for propagation of the information state. 


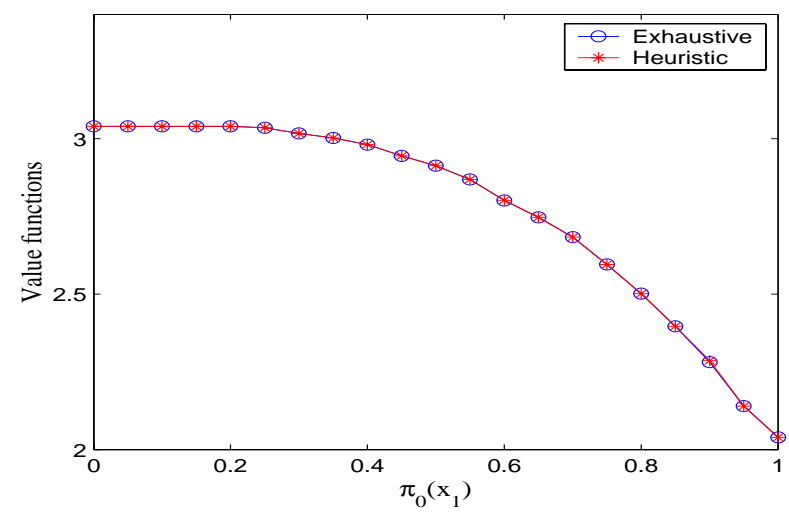

Fig. 1. Comparison of value functions obtained through the exhaustive search and the heuristic algorithm.

The concept of mutual information [8] is useful here. The mutual information $I(W ; V)=H(W)-H(W \mid V)$ between two random variables $W$ and $V$ reveals how much information of $W$ is contained in $V$ or vice versa. Here $H(\cdot)$ and $H(\cdot \mid \cdot)$ denote the entropy and the conditional entropy, respectively. In the setup of this paper one can compare $I\left(X_{i+j-1} ; Y_{n}\right)$ for $i \leq n \leq i+j-1$.

Lemma 4.1: For $n_{1} \leq n_{2} \leq n_{3}$,

$$
I\left(X_{n_{3}} ; Y_{n_{2}}\right) \geq I\left(X_{n_{3}} ; Y_{n_{1}}\right),
$$

i.e., the mutual information between the state and the observation decreases as their time difference increases.

Sketch of proof. To illustrate the idea, consider the case $S=M=2$ (two-state and two-output), and without loss of generality, we shall show $I\left(X_{1} ; Y_{1}\right) \geq I\left(X_{1} ; Y_{2}\right)$ (this is equivalent to proving $I\left(X_{2} ; Y_{2}\right) \geq I\left(X_{2} ; Y_{1}\right)$ since the reverse chain is also Markovian).

The conditional PMF of $Y_{1}$ given $X_{1}$ is given by $C=\left[c_{i k}\right]$, while that of $Y_{2}$ given $X_{1}$ is given by $C^{\prime}=$ $\left[c_{i k}^{\prime}\right]=A^{T} C$, where $A=\left[a_{i j}\right]$ is the probability transition kernel. This implies $c_{11}^{\prime}, c_{21}^{\prime} \in\left[c_{11}, c_{21}\right]$ (assuming $c_{11} \leq$ $\left.c_{21}\right)$. Hence $Y_{2}$ tends more to produce same outcomes for different states $X_{1}$ than $Y_{1}$ does, implying $I\left(X_{1} ; Y_{1}\right) \geq$ $I\left(X_{1} ; Y_{2}\right)$.

As an illustration, Fig. 2 compares $I\left(X_{2} ; Y_{1}\right), I\left(X_{2} ; Y_{2}\right)$, and $I\left(X 2 ; Y_{1}, Y_{2}\right)$, where the horizontal axis is $\operatorname{Pr}\left(X_{1}=\right.$ $\left.x_{1}\right)$. The transition kernel used here is $A\left(u_{1}\right)$ in (8), and

$$
C=\left[\begin{array}{lll}
5 / 9 & 1 / 3 & 1 / 9 \\
1 / 9 & 1 / 3 & 5 / 9
\end{array}\right] .
$$

A problem for $S=2, B=2$, and $M=3$ is solved using the exhaustive search. The matrices $A\left(u_{1}\right)$ and $A\left(u_{2}\right)$ are as in (8), $C$ as in (12), and $G$ as in (10). Blocks of both length 1 and length 2 show up in the optimal quantization schemes. A closer look reveals that there are two coding schemes for data blocks of length 2, both partition $\mathcal{Y}^{2}$ into two sets:

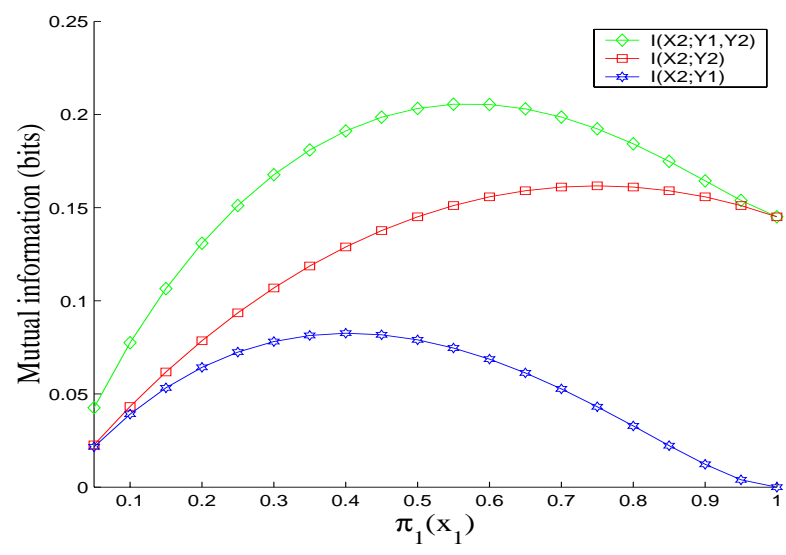

Fig. 2. Comparison of mutual information.

- Scheme 1: $\left[\left(y_{1}, y_{1}\right),\left(y_{2}, y_{1}\right),\left(y_{3}, y_{1}\right)\right], \quad$ and $\left[\left(y_{2}, y_{1}\right),\left(y_{2}, y_{2}\right),\left(y_{2}, y_{3}\right),\left(y_{3}, y_{1}\right),\left(y_{3}, y_{2}\right),\left(y_{3}, y_{3}\right)\right]$;

- Scheme 2: $\left[\left(y_{1}, y_{1}\right),\left(y_{2}, y_{1}\right),\left(y_{3}, y_{1}\right),\left(y_{2}, y_{1}\right)\right]$, and $\left[\left(y_{2}, y_{2}\right),\left(y_{2}, y_{3}\right),\left(y_{3}, y_{1}\right),\left(y_{3}, y_{2}\right),\left(y_{3}, y_{3}\right)\right]$.

Here each pair $\left(y_{m}, y_{l}\right)$ represents an outcome of $\left(Y_{i}, Y_{i+1}\right)$. It can been seen that in both schemes, $Y_{i+1}$ is quantized more finely than $Y_{i}$.

\section{Numerical Results on Tradeoff Analysis}

Fig. 3 shows the running cost $J^{r} v s$ the total bits $J^{c}$ as the weighting coefficient $\lambda$ (communication cost per bit) is varied from 0.01 to 0.5 , calculated using the heuristic method. Here $S=2, M=3, K=2, B=2, N=2$, and $A(u)$, $G$, and $C$ are as in (8), (10), and (12) respectively. Higher $\lambda$ leads to less bits and consequently higher running cost. As $\lambda$ changes from 0 to $\infty$, the resulting optimal solutions for (1) constitute the family of Pareto optimal solutions for the two-objective problem, providing the tradeoff curve between the running cost and the communication bits.

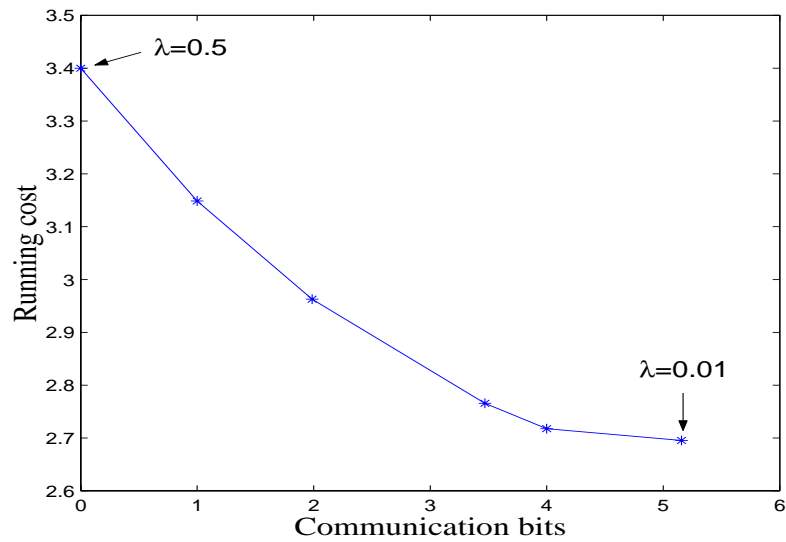

Fig. 3. Running cost vs. the communication bits.

In general the optimal quantization schemes involve timevarying block lengths, and for different a priori PMFs of $X_{0}$, the total communication bits might be different. Next 
we restrict to the class of quantization/control schemes that carry certain fixed block-length $B_{0}$, and compare the control performance of optimal schemes in this class as $B_{0}$ is varied while the total number of bits is fixed. The motivation is to see, given a fixed number of communication bits, which one leads to better control performance: more frequent communication with coarser quantization, or less frequent communication with finer quantization. In the examples here, we compare the case $B_{0}=1$ (Block-1) with the case $B_{0}=2$ (Block-2). In Block-1, 1 bit is allocated for each block; in Block-2, two bits are allocated for each block. The time horizon $N=4, S=2, M=3, K=2$. Two sets of parameters are used. In the first set, $A(u), C$, and $G$ are as in (8), (12), and (10), respectively. In the second set,

$$
\begin{aligned}
& A\left(u_{1}\right)=\left[\begin{array}{ll}
1 / 5 & 3 / 5 \\
4 / 5 & 2 / 5
\end{array}\right], A\left(u_{2}\right)=\left[\begin{array}{ll}
5 / 7 & 3 / 7 \\
2 / 7 & 4 / 7
\end{array}\right], \\
& C=\left[\begin{array}{lll}
3 / 16 & 9 / 16 & 4 / 16 \\
8 / 25 & 7 / 25 & 10 / 25
\end{array}\right], G=\left[\begin{array}{ll}
5.4 & 2.8 \\
3.5 & 5.9
\end{array}\right] .
\end{aligned}
$$

For the first set of parameters, control performance is better under Block-1 (Fig. 4). For the second set of parameters, Block-1 does better than Block-2 except for some initial conditions (Fig. 5). The figures suggest that, for the current setup, there is no general answer about which (fixed) blocklength offers the best control performance; indeed, the optimal block-length seems to depend on the parameters and the initial condition.

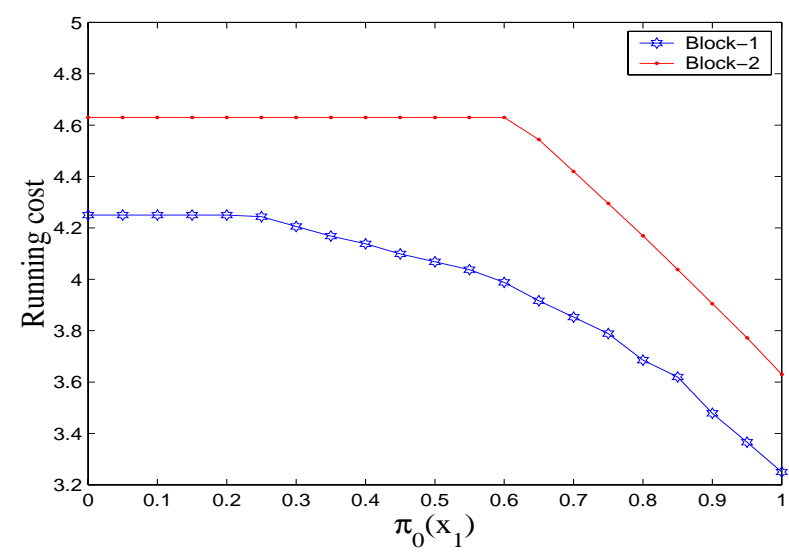

Fig. 4. Running cost vs. $\operatorname{Pr}\left(X_{0}=x_{1}\right)$ based on the first set of parameters.

\section{CONCLUSIONS}

In this paper the problem of combined quantization and control with block-coding has been studied, where the DP equation is of a special form. A heuristic algorithm has been proposed to facilitate solving the DP equations numerically. The algorithm lifts the output observation from the discrete space $\mathcal{Y}^{j}$ to the space of conditional PMFs of the state thus endowing a metric on $\mathcal{Y}^{j}$.

An important goal of this paper has been to understand the impact of block-coding on the control performance. It

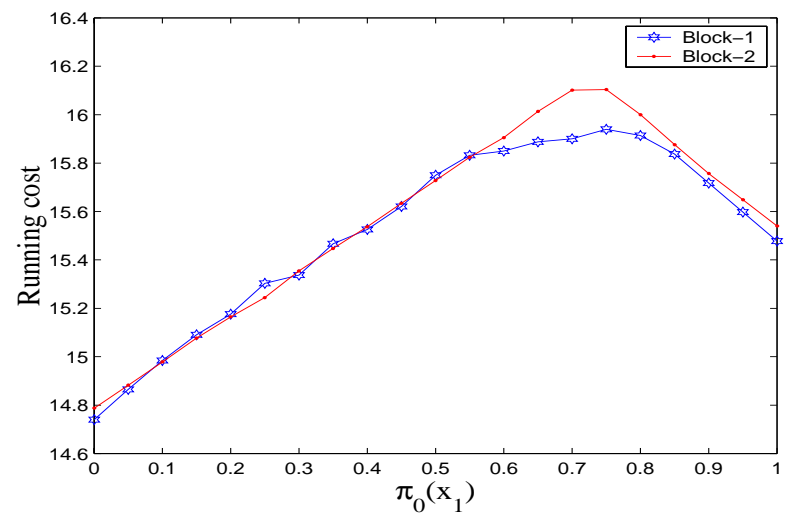

Fig. 5. Running cost vs. $\operatorname{Pr}\left(X_{0}=x_{1}\right)$ based on the second set of parameters.

has been found that the more recent observation is more relevant for the purpose of control, and indeed the optimal scheme quantizes it more finely. Numerical results have been presented showing the tradeoff between the control performance and the communication needs.

Despite the significant speedup it provides comparing with the exhaustive search algorithm, the heuristic algorithm is still of combinatorial nature and its complexity grows exponentially with the number $M$ of outcomes. However, the current framework lends itself naturally to various stochastic and deterministic tools in the literature of vector quantization (VQ) [10]. Future work will involve applying VQ algorithms to develop scalable methods for solving the DP equations.

\section{REFERENCES}

[1] W. S. Wang and R. W. Brockett, "Systems with finite communication bandwidth constraints - II: stabilization with limited information feedback," IEEE Trans. Automat. Control, vol. 44, no. 5, pp. 1049$1053,1999$.

[2] S. Tatikonda, Control under Communication Constraints, Ph.D. thesis, M.I.T., 2000.

[3] N. Elia and S. K. Mitter, "Stabilization of linear systems with limited information," IEEE Trans. Automat. Control, vol. 46, no. 9, pp. 1384 1400, 2001.

[4] D. Hristu-Varsakelis and P. R. Kumar, "Interrupt-based feedback control over a shared communication medium," in Proceedings of the 41st IEEE Conference on Decision and Control, Las Vegas, NV, 2002, pp. pp. 3223-3228.

[5] D. Liberzon, "Stabilizing a nonlinear system with limited information feedback," in Proceedings of the 42nd IEEE Conference on Decision and Control, Maui, Hawaii, 2003, pp. 182-186.

[6] V. S. Borkar, S. K. Mitter, and S. Tatikonda, "Optimal sequential vector quantization of Markov sources," SIAM J. Control Optim., vol. 40, no. 1, pp. 135-148, 2001.

[7] V. S. Borkar, S. K. Mitter, and S. Tatikonda, "Markov control problems under communication constraints," Communications in Information and Systems, vol. 1, no. 1, pp. 15-32, 2001.

[8] T. M. Cover and J. A. Thomas, Elements of Information Theory, John Wiley \& Sons, Inc, New York, 1991.

[9] J. S. Baras, X. Tan, and W. Xi, "Jointly optimal quantization, estimation, and control of hidden Markov chains," in Proceedings of the 42nd IEEE Conference on Decision and Control, Maui, Hawaii, 2003, pp. 1098-1103.

[10] A. Gersho and R. M. Gray, Vector Quantization and Signal Compression, Kluwer Academic Publishers, 1992. 